\section{Are We Doing Enough to Contain Acinetobacter Infections?}

\section{To the Editor:}

Nosocomial infections caused by antibiotic-resistant strains of Acinetobacter are being reported with increasing frequency and are a major source of concern. Many strains have a high level of resistance to multiple antimicrobials and are associated with a high mortality rate, especially for pneumonia and bloodstream infections. ${ }^{1}$ Risk factors associated with infection have been reported to include intubation, prolonged stay in the intensive care unit and hospital, prior use of broad-spectrum antibiotics, ${ }^{2}$ mechanical ventilation, ${ }^{3}$ prior surgery, and urinary catheterization. ${ }^{4}$

Intensive efforts have been applied to preventing or containing outbreaks caused by Acinetobacter. The Centers for Disease Control and Prevention $(\mathrm{CDC})$ recommendations regarding control of multidrugresistant gram-negative rods (including Acinetobacter) suggest that, in addition to Standard Precautions, Contact Precautions should be used for infected or colonized patients. ${ }^{5}$ However, despite application of these recommendations, nosocomially acquired Acinetobacter remains problematic, resulting in substantial associated morbidity and mortality, higher treatment costs, and prolonged hospital stay.

It has been reported previously that Acinetobacter may be spread by the airborne route. ${ }^{6,7} \mathrm{~A}$ recent report ${ }^{8}$ supports this idea, based on observation that outbreaks of resistant Acinetobacter occurred in two facilities in which the index case was placed on Contact Precautions. Sedimentation plates yielded Acinetobacter both inside and outside of the infected patient's room. In contrast, no crosstransmission was observed in the facility where the index case was placed on Airborne Precautions.

We also have investigated the potential for multidrug-resistant Acinetobacter to spread by the droplet and airborne route in seven patients with respiratory tract infection or colonization.

Sedimentation plates were placed within a patient's room at measured intervals from the patient. The percentage of sedimentation plates with Acinetobacter colonies at various distances from the patient were: $1 \mathrm{ft}$, $42 \% ; 3 \mathrm{ft}, 28 \% ; 5 \mathrm{ft}, 75 \% ; 7 \mathrm{ft}, 60 \% ; 9 \mathrm{ft}$, $57 \%$; and $11 \mathrm{ft}, 40 \%$ (maximum spatial separation achievable within the room). In several instances, Acinetobacter was also detected on sedimentation plates placed outside of the patient's room and as far away as the nursing station (approximately $22 \mathrm{ft}$ from the room). Strains isolated from the patient's respiratory cultures and from sedimentation plates had the same antibiogram.

The detection of Acinetobacter in all areas within the rooms tested, and beyond, suggests a potential for airborne dissemination, as well as for droplet dissemination (which would be confined to a distance of approximately $3 \mathrm{ft}$ from the patient).

Considering the continuing difficulty in controlling the spread of Acinetobacter throughout our healthcare facilities, these reports and findings, which suggest the potential for airborne transmission of Acinetobacter, are troubling, since current practice, based on CDC guidelines, ${ }^{5}$ does not specifically address the potential for droplet or airborne transmission of Acinetobacter.

The potential for droplet and airborne transmission must be further evaluated with appropriately designed and controlled studies before any recommendation regarding the widespread use of these enhanced precautions can be considered. However, limited use of airborne precautions for pan-resistant strains of Acinetobacter infecting or colonizing the respiratory tract might be prudent for selected cases. This would especially pertain to patients with active cough or on mechanical ventilation requiring frequent suctioning.

\section{REFERENCES}

1. Seifert H, Strate A. Pulverer G. Nosocomial bacteremia due to Acinetobacter baumannii. Clinical features, epidemiology and predictors of mortality. Medicine (Baltimore) 1995;74:340-349.

2. Scerpella EG, Wanger AR, Armitige L, Anderlini P, Ericsson CD. Nosocomial outbreak caused by a multi-resistant clone of Acinetobacter baumannii: result of the case control and molecular epidemiologic investigations. Infect Control Hosp Epidemiol 1995;16:92-97.

3. Cox TR, Roland WE, Dolan ME. Ventilatorrelated Acinetobacter outbreak in an intensive care unit. Mil Med 1998;163:389-391.

4. Gomez J, Simarro E, Banos V, Requena I. Ruiz J, Garcia F, et al. Six year prospective study of risk and prognostic factors in patients with nosocomial sepsis caused by
Acinetobacter baumannii. Eur $J$ Clin Microbiol Infect Dis 1999;18:358-361.

5. Garner JS, the Hospital Infection Control Practices Advisory Committee. Guidelines for isolation precautions in hospitals. Infect Control Hosp Epidemiol 1996;17:53-80.

6. Allen KD, Green HT. Hospital outbreak of multi-resistant Acinetobacter anitratus: an airborne mode of spread? I Hosp Infect 1987;9:110-119.

7. Jawad A, Heritage J, Snelling AM, GascoyneBinzi DM, Hawkey PM. Influence of relative humidity and suspending menstrua on survival of Acinetobacter spp on dry surfaces. $J$ Clin Microbiol 1996;34:2881-2887.

8. Bernards AT, Frenay HME, Lim BT, Hendriks, WDH, Dijkshoorn L, van Boven CPA. Methicillin-resistant Staphylococcus aureus and Acinetobacter baumannii: an unexpected difference in epidemiologic behavior. Am J Infect Control 1998;26:544551.

Steven E. Brooks, PhD Mary A. Walczak, RN, CIC Rizwanullah Hameed, MD Kingsbrook Jewish Medical Center Brooklyn, New York

\section{Epidemiology of Nosocomial Infections at Fukuoka University Hospital}

\section{To the Editor:}

To investigate the epidemiology of nosocomial infections at Fukuoka University Hospital (850-bed), hospitalwide surveillance was conducted from June 1995 to March 1996. The emergency center, the neonatal intensive care unit, and all of the inpatient wards, except the psychiatry ward, were included.

Based on attending physician's reports, bacteriology reports, patient charts, and clinical ward rounds, nosocomial infections were determined by the infection control team (ICT) according to Centers for Disease Control and Prevention definitions. ${ }^{1}$ The bacteriology reports were made and prepared by the ICT just on all methicillin-resistant Staphylococcus aureus (MRSA) isolates and isolates from blood cultures. The ICT used patient records to determine whether reported cases represented infection or colonization. Once weekly, the ICT made clinical ward rounds to each unit.

Nosocomial infection rates were calculated by dividing the number of nosocomial infections by the number 